\title{
Magnetically controlled ballistic deposition. A model of polydisperse granular packing
}

\author{
K. Trojan ${ }^{\mathrm{a}, \mathrm{b}, *}$, M. Ausloos ${ }^{\mathrm{b}}$ \\ a Institute of Theoretical Physics, University of Wroctaw, pl. M. Borna 9, PL 50-204 Wroctaw, Poland \\ ${ }^{\mathrm{b}}$ SUPRAS and GRASP Institute of Physics ${ }^{1}$, B5, University of Liège, B-4000 Liège, Belgium
}

Received 5 February 2003

\begin{abstract}
The flow and deposition of polydisperse granular materials is simulated through the magnetic diffusion limited aggregation (MDLA) model. The random walk undergone by an entity in the MDLA model is modified such that the trajectories are ballistic in nature, leading to a magnetically controlled ballistic deposition (MBD) model. This allows to obtain important ingredients about a difficult problem that of the nonequilibrium segregation of polydisperse sandpiles and heterogeneous adsorption of a binary distribution of particles which can interact with each other and with an external field. Our detailed results from many simulations of MBD clusters on a two-dimensional triangular lattice above a flat surface in a vertical finite size box for binary systems indicates intriguing variations of the density, "magnetization", types of clusters and fractal dimensions. We derive the field and grain interaction-dependent susceptibility and compressibility. We deduce a completely new phase diagram for binary granular piles and discuss its complexity inherent to different grain competition and cluster growth probabilities.
\end{abstract}

(c) 2003 Elsevier B.V. All rights reserved.

PACS: 45.70.Cc; 61.43.Hv; 07.05.Tp; 81.05.Rm; 61.43.Gt

Keywords: Sandpile; Kinetic growth model; Ballistic DLA; Density; Magnetization; Susceptibility; Compressibility; Fractal dimension

\footnotetext{
* Corresponding author.

E-mail address: ktrojan@ift.uni.wroc.pl (K. Trojan).

${ }^{1}$ GRASP $=$ Group for Research in Applied Statistical Physics; SUPRAS = Services Universitaires Pour la Recherche et les Applications en Supraconductivité.
} 


\section{Introduction}

Understanding the flow and static structures of granular matter is becoming increasingly relevant. Many raised questions are tackled along various lines of approach [1]. It has already been claimed that the simplifications found in basic models need to be improved in order to explain features of such complex materials. Granular pile spreading processes driven by cooperative nonlinear evolution rules lead to developed patterns which often reach a high level of complexity. It is of present interest to examine whether growth models can be used for describing granular structures and related material properties. Furthermore, the nonlinear processes at work in granular flows and depositions hint toward simulation approaches [2]. Cooperative effects in ballistic deposition of hard disks have been recently mentioned [3].

Kinetic growth models (KGM) have received much attention [4] like the Eden model [5] and the diffusion limited aggregation model [6] (DLA). They have served to describe nonequilibrium phenomena like film or crystal growth [7,8], epidemics [9], material fractures [10], etc. In all cases, such models are mainly concerned by the transition from dense branching to dendritic morphology.

One important physical constraint has to be considered in describing granular materials: the materials are not made of symmetrical (spherical or cubic) entities. The surface of grains is usually rough, thus leading to specific angles of repose [11]. Also, the grain anisotropy leads to phenomena like jams [12], in flow, and arches [13], in static structures. It seems therefore necessary to have at least one degree of freedom in order to describe grains; we are even aware that only one degree is a very strong approximation. This degree of freedom should be coupled to some field, just like a spin to a magnetic field. Whence one can imagine that grains are identical entities except for one degree of freedom, call it a spin though it can be any physical feature of particular interest, like the grain roughness or shape feature. Clearly, a spin allows for referring to a direction or a rotation process; if this is admitted, to take such a degree of freedom into account in describing granular piles should basically improve the granular state overall description. (Generalizations are immediately imagined by anyone familiar with spin models and statistical mechanics; one can later on imagine many component vector models, including Potts-like models [14].) In fact, a constrained Ising spin chain has been recently considered and studied as a toy model for granular compaction [15]. The exchange energy $J$ describing the "spin-spin interaction" is analogous in granular matter to the contact energy due to surface roughness between grains. A similar interpretation of $J$ for flows can be found in Pandey et al. [2]. The external "magnetic" field in such a case can be, e.g. a wind field, the sign depending on, e.g. change in pressure due to grain drag. We thus combine topology and mass (or weight) in order to describe granular materials in a simple way.

The above ideas remind us that a similar set of considerations has been found in the magnetic Eden model [16] and in the magnetic diffusion limited aggregation (MDLA) model [17] when attempting to describe crystal growth in a magnetic field, when there is a competition between entities. Aggregation can proceed under short-range or long-range dipolar interactions in fact $[18,19]$. However, the studies pertained to the growth of clusters starting from a point seed. In a recent set of investigations on the 
magnetic Eden model, Albano et al. [20-23] have pointed out the interest of such models for examining deposition and film growth, thus starting from a substrate- sometimes with rather complex realistic rules. The studies are also related to nonequilibrium wetting questions [24], -and other deposition problems [25].

The same type of studies can be done with the MDLA, i.e. examining the growth of clusters from a substrate. It is clear that a substrate having finite (or not) size destroys the spatial isotropy or more generally spatial symmetry. In simple words, there is a top and bottom, if the substrate is horizontal. It is obviously of interest for granular materials in a gravitational field to consider what happens only in the half space above the (finite size of course) substrate. This reduction is however of fundamental and practical interest because one can also consider that the system is in a vertical box with walls, as in the Albano et al. film growth geometry [21-23] with binary competing entities. In DLA, the diffusing particle follows a random walk [6]. However, it seems very hard to let this usual DLA rule holds here concerning the path of the granular entity launched far away from the seed or substrate. In the present considerations, it seems more appropriate to let the granular entity follows a ballistic vertical trajectory like in rain models [25] rather than a random walk.

In this paper, we calculate what changes result in the features of a classical ballistic deposition [25-27] (BD) model when we add one extra degree of freedom, a "spin", to the classical BD. The spin reflects, e.g., the orientation of oblate grains or their roughness characteristic. The spins, as usual, interact through some exchange energy $J$ which, for grains, is often mechanical or electrostatic in nature. The external field $H$ is thought to be the image of a classical field positioning or influencing the flow of grains, like some wind velocity or, more generally, pressure difference, or an electric field. This model is hereby called the magnetically controlled ballistic deposition (MBD) model falls into the category of kinetic growth models. In Section 2, we enumerate the algorithm rules and briefly comment upon them. In Section 3 we present results on the "density" and "magnetization", "susceptibility" and "compressibility". The types of clusters and their fractal dimensions are discussed in Section 4. In Section 5, a brief conclusion can be found.

\section{Experimental procedure}

For simplicity we will thereafter call a grain, a spin. It will take here only two states (up or down) or two values $(+1$ or -1$)$. The external field is supposed to be constant and uniform throughout the whole system. For obvious reasons, like higher packing considerations and possible geometrical frustrations, the underlying lattice should not have a square symmetry; we have taken a triangular lattice in the following simulations (Fig. 1).

One of the main problems to be tackled is how to choose the best rule for aggregating granular falling entities (spins) in order to get them stick together and form clusters. We have chosen the simplest sticking rule, usual in statistical physics, i.e., namely a Metropolis-like rule, as also in Pandey et al. [2], presented here below. The algorithm 

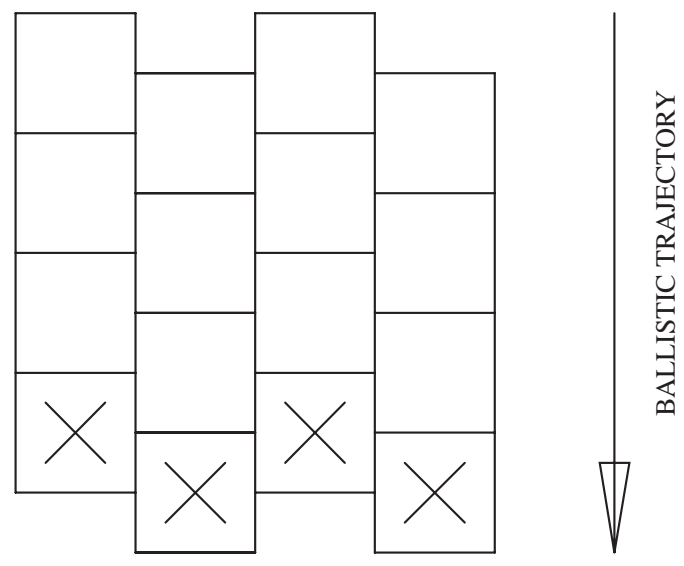

Fig. 1. Example of a $4 \times 4$ triangular lattice. The arrow indicates the fall direction. The substrate has been marked with crosses.

goes as follows:

1. first, we choose a horizontal substrate of spins with a predetermined (for example antiferromagnetic-like or random) configuration;

2. a falling (up or down) spin is dropped along one of the lattice lines (see Fig. 1) from a height $r_{\max }+5 a$, where $r_{\max }$ is the largest distance between a cluster site and the substrate, i.e., here it is the height of the highest column growing from the substrate on the lattice; at each step down the spin can flip, i.e., change its sign, with equal probability; e.g. the anisotropic grain can rotate;

3. the spin goes down until it reaches a site perimeter of the cluster; the local gain in the Ising energy

$$
\beta E=-\beta J \sum_{\langle i, j\rangle} \sigma_{i} \sigma_{j}-\beta H \sum_{i} \sigma_{i},
$$

is calculated before and after the spin possible impact, thus cluster growth. If the gain is negative the spin sticks to the cluster immediately (sticking probability $=1.0$ ) and we go back to step (2). In the opposite case the spin sticks to the cluster with a rate $\exp (-\Delta \beta E)$ where $\Delta \beta E$ is the local gain in the Ising energy. If the spin does not stick to the cluster it continues going down toward the substrate or bottom of the box. Of course if the site just below the spin is occupied the spin immediately stops and sticks to the cluster. When the spin sticks to the cluster we go back to step (2).

4. After dropping a (large) number of spins the physical quantities of interest like the magnetization, density, fractal dimension, etc. are computed.

It should be noticed that there is no toppling nor relaxation at this time like in Manna sandpile model or its extensions [15,28,29]. Moreover, since the number of nearest neighbors on a triangular lattice is equal to 6 , and due to the rule of MBD 
Table 1

Rate of sticking and configurations in the MDM model on the triangular lattice. First (second) column shows the rate of sticking an up (down) spin to the preexisting spin configuration

\begin{tabular}{l|l|l|}
\hline Spin up & Spin down \\
$\exp (+\beta H)$ & $\exp (+\beta J-\beta H)$ \\
$\exp (-\beta J+\beta H)$ & $\exp (+2 \beta J-\beta H)$ \\
$\exp (-\beta J+\beta H)$ & $\exp (-2 \beta J-\beta H)$ \\
$\exp (+2 \beta J+\beta H)$ & $\exp (+3 \beta J-\beta H)$ \\
$\exp (-3 \beta J+\beta H)$ & $\exp (-3 \beta J-\beta H)$ \\
$\exp (+4 \beta J+\beta H)$ & $\exp (+4 \beta J-\beta H)$
\end{tabular}

(above paragraph), we do not have to take into considerations the spin configurations in which the depositing spin has a neighbor just vertically over it or just below (because it would then always stick to the cluster). Therefore, there are only four neighboring sites where spin configurations are relevant for calculating the local gain in the Ising energy. On the other hand there are three kinds of site occupation: spin up, spin down and no spin, hence 52 configurations (excluding the empty one-when there is no spin on the perimeter). Some of these configurations are symmetrical with respect to rotations. Finally, 23 configurations are to be examined having at least one spin on the perimeter. All these sticking configuration rates (also distinguishing the sign of the falling spin) are shown in Table 1. The contribution to the sticking rate arising from the interaction of the depositing spin with the field has also to be evaluated. The rates of sticking to the cluster read like

$$
P_{n, s}=\mathrm{e}^{-\Delta \beta E}=\mathrm{e}^{s(n \beta J+\beta H)},
$$

where $\triangle \beta E$ is the local gain of the Ising energy, and $n$ is the difference between the number of up and down spins: the possible values are $-4,-3, \ldots, 4$, and $s$ is the value (or sign) of the falling $\operatorname{spin}(-1$ or 1$)$. Equating all these rates lead us to a set of 16 relations between $\beta J$ and $\beta H$; in fact as in the MDLA [17]. Fig. 2 determine 32 regions where granular packing cluster growth processes differ from each other. The 


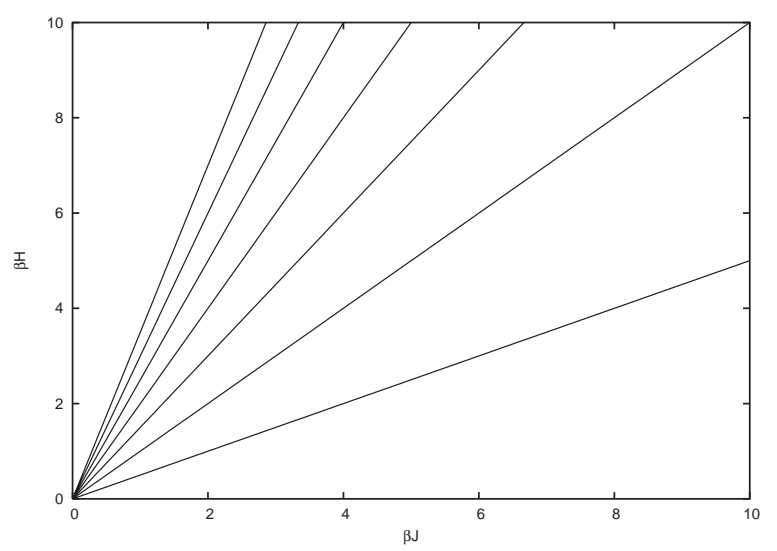

Fig. 2. Relations between $\beta J$ and $\beta H$ on the $\beta J-\beta H$ plane; 32 regions result from these relations.

case $\beta J=0$ and $\beta H=0$ corresponds to the usual ballistic deposition model (standard deposition without spin and field) [4].

Notice that the model contains two order parameters, as in the Blume-EmeryGriffiths model [30,31], one corresponds to the density, the other to the magnetization.

\section{Numerical results and discussion}

All results reported below are for a triangular lattice of horizontal size $L=100$, i.e., the width of the seed substrate, and when the pile made of clusters has reached a 500 lattice unit height. Every reported data point corresponds to an average over 1000 simulations.

\subsection{Density}

We define the density of a cluster as

$$
G=\frac{\text { number of spins in the cluster }}{\text { number of sites on the lattice }}
$$

in which obviously the number of lattice sites $=50000$. Fig. 3 illustrates the behavior of the density with respect to the $\beta H$ parameter. This figure convinces us that the results are symmetrical with respect to $\beta H=0$. Therefore, in the following subsections we will often present results for $\beta H>0$ only. It is observed that the density presents a sharp minimum when $\beta H= \pm \beta J$. The granular pile is rather loosely packed since the density varies between 0.37 and 0.45 . This low value with respect to experimental findings arises from the fact that we have not included relaxation processes in this investigation.

In Fig. 4 one illustrates in a 3D way the influence of the interaction part of the hamiltonian, i.e., $\beta J$, on the density. This sort of diagram allows us to emphasize that 


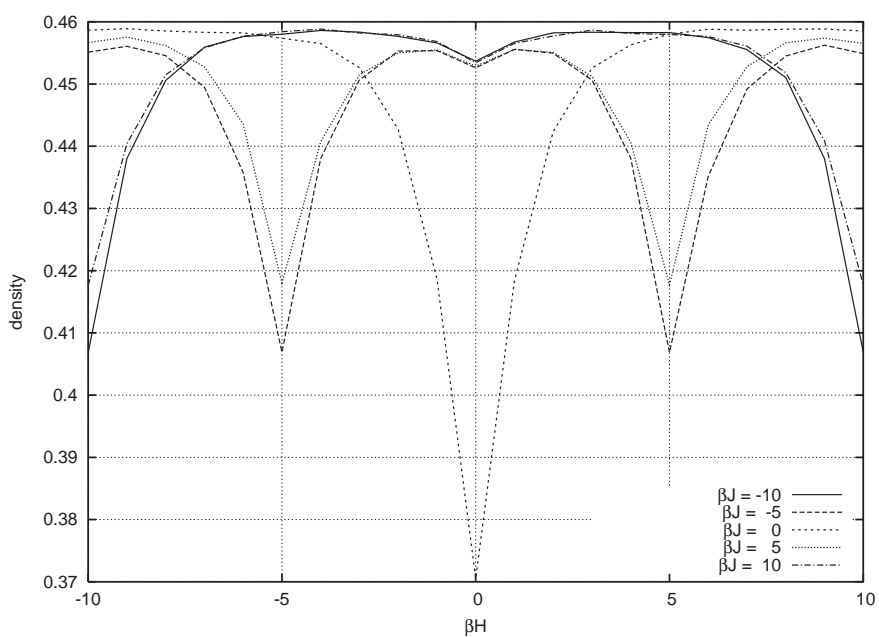

Fig. 3. Dependence of density on $\beta H$ for different $\beta J$. Observe that the behavior is symmetrical with respect to $\beta H=0$.

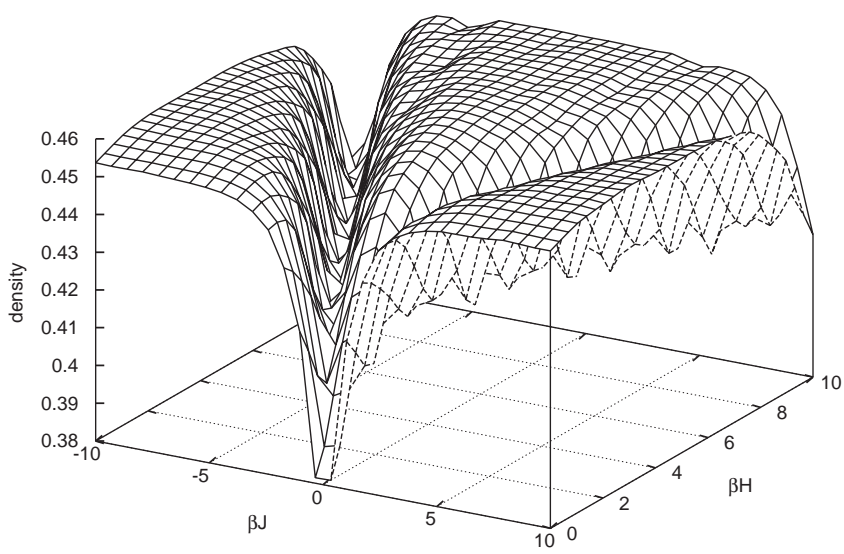

Fig. 4. Dependence of the MBD pile density on $\beta H$ and $\beta J$. Observe trenches at borders between plateau regions.

the density is almost the same everywhere, as mentioned above, but there are several minima: the main density variations occur along lines bordering plateau regions, lines which correspond to the equal probability lines mentioned in the previous section. One can observe a set of trenches near these borders between different growth (or packing) regions. In these trenches, the density is markedly lower than in the immediate neighborhood. Indeed such trenches correspond to the highest possible rate of sticking, thus to a condition for loose packing (see also below). 


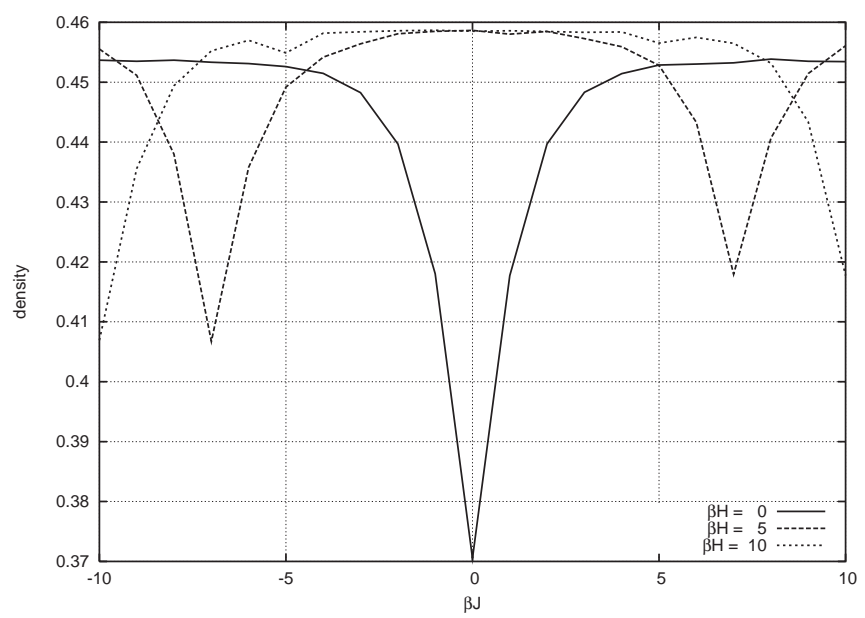

Fig. 5. Dependence of the density on $\beta J$ for different $\beta H$.

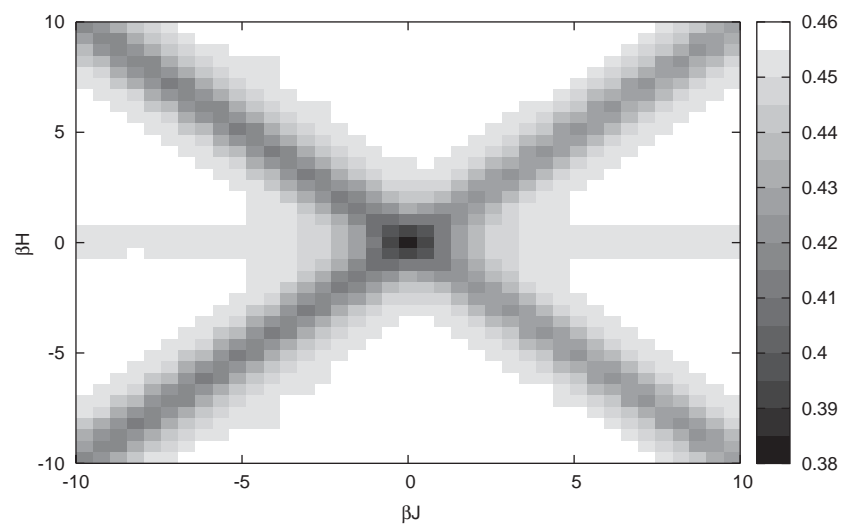

Fig. 6. Dependence of the density on $\beta H$ and $\beta J$. Different gray colors correspond to different density levels with "color" scale indicated on the right.

From Fig. 5, the value of the density is seen to be slightly different for $\beta J>0$ and for $\beta J<0$, but remains qualitatively the same. These differences indicate that a little higher density is obtained for ferromagnetic systems $\beta J>0$ than for antiferromagnetic ones, in particular when the external field is different from 0 . Something similar had been found in studies on the MDLA [17]. Further discussion on this point is postponed for after examination of the clusters in Section 4.

Another illustration of the density dependence is exhibited in Fig. 6 as a projection on the $(\beta J, \beta H)$ plane. The main trenches are observed, i.e., only 10 , out of all 32 possible ones: they are located at $\beta H=0, \beta H= \pm \beta J, \beta H= \pm 2 \beta J$. One can distinguish a trench for positive and negative values of $\beta J$ and $\beta H$. The last one is not clearly visible in Fig. 6, but in Fig. 5, one can observe a small hollow near the trench $\beta H=-\beta J$ for 
(a)

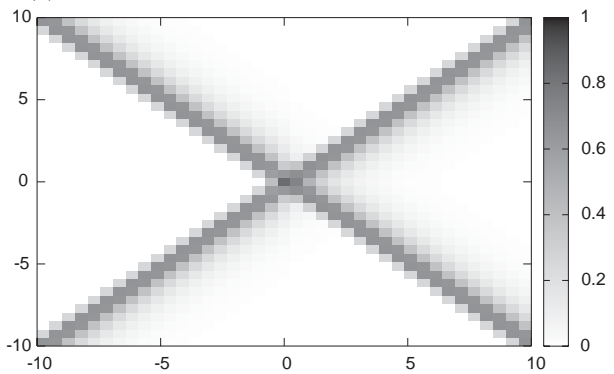

(c)

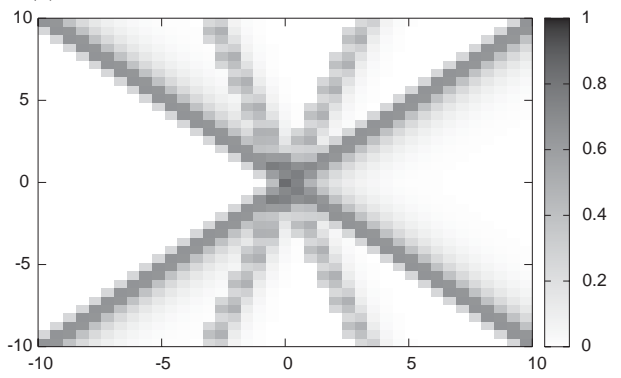

(b)

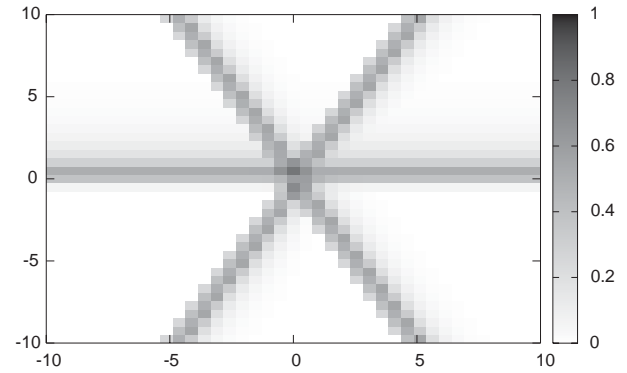

(d)

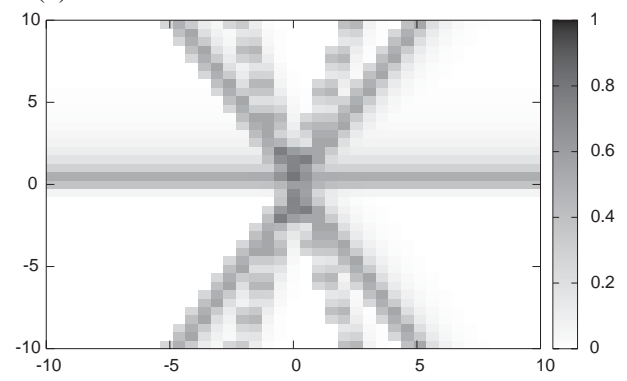

Fig. 7. Theoretical maximum probability of sticking a spin to a cluster for a cluster configuration with: (a) one spin, (b) two spins, (c) three spins, (d) four spins.

$\beta J<0$. Hollows are positioned along the trench $\beta=-2 \beta J$. Such structures (hollows) are artefacts due to resolution of the simulation.

The $\beta H= \pm 2 \beta J$ trench is very clearly seen in Fig. 5; for $\beta H=5$, a small minimum is observed for $\beta J= \pm 5$, a symptom of the existence of a trench.

To explain the behavior of the density due to $\beta J$ and $\beta H$ parameters, let us compute the maximum probability of cluster sticking for the configuration having $n$ spins in a site neighborhood. The results for each configuration, with respect to the number of spins in the perimeter are presented in Fig. 7. The discrete color changes is real but the zig zag blurring is an artefact due to the numerical sampling with $\beta J$ and $\beta H$. Next, from this figure it can be concluded that the biggest drop in density occurs for $\beta H= \pm \beta J$ indeed (Fig. 7a). The reason stems from the configurations which correspond to a case with a small amount of spins, i.e., with one or three spins, thus to a possibly high relative change in Ising energy. This observation further explains the existing density trenches observed in Fig. 4 (or Fig. 6) for $\beta H= \pm \beta J$.

A very similar conclusion pertaining to the $\beta H=0$ and $\beta H= \pm 2 \beta J$ lines can be drawn for the appropriate trenches in Fig. 4 (or Fig. 6); the drop is thereby smaller because these configurations contain two and four spins-starting from a four spin configuration does not obviously reduce the density after one extra spin sticks to the cluster.

At each step, the available "volume of interest" on the perimeter corresponds to seven sites. For a configuration with the largest possible number of "useful" spins for 


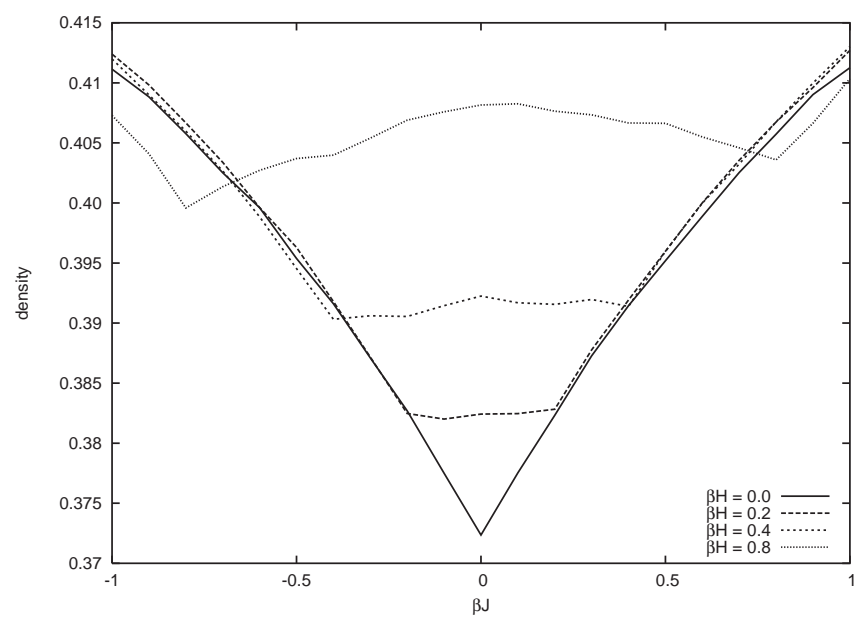

Fig. 8. Dependence of the density on $\beta J$ for different $\beta H$.

sticking in the perimeter we have a final five spin configuration $(4+$ the dropping spin), whence the local density is at most $\frac{5}{7} \approx 0.71$. However, this occurs at most six times (out of 23 ), see Table 1 leading to a rough estimate of $2(6 / 23)(5 / 7)=0.37$ for the density.

On the other hand, configurations with three and four spins on the perimeter lead to an increase in the density after spin sticking roughly equal to 0.46 as observed.

The behavior of the density in the vicinity of the zero field and zero interaction case is of interest with respect to observe deviations from the classical BD model. In Fig. 8, it is seen that when the field is different from 0 the density is almost constant over some $\beta J$ interval, the width of which depending on the field value, i.e., the higher the field, the wider the interval. Observe that the density value in the interval does not seem to be varying linearly with the field. Notice that the density dependence observed in Fig. 5 and its characteristic structures, i.e., the trenches are again observed for moderately high fields (ca. 0.8).

Finally, Fig. 9 exhibits the behavior of the density for $\beta H=\beta J$, i.e., along the deepest trench (Fig. 4 or Fig. 6). It should be emphasized that the $\beta J<0$ trench level has a lower density than the $\beta J>0$ one due to the imbalance in sticking probabilities for preferred ferromagnetic or antiferromagnetic-like configurations. Worth mentioning is that the second deepest trench, i.e., $\beta H=-\beta H$ has exactly the same dependence like that shown in Fig. 9.

\subsection{Magnetization}

In this subsection we present results concerning the magnetization of the cluster of spins. The magnetization is defined as

$$
M=\frac{n_{+}-n_{-}}{n_{+}+n_{-}},
$$




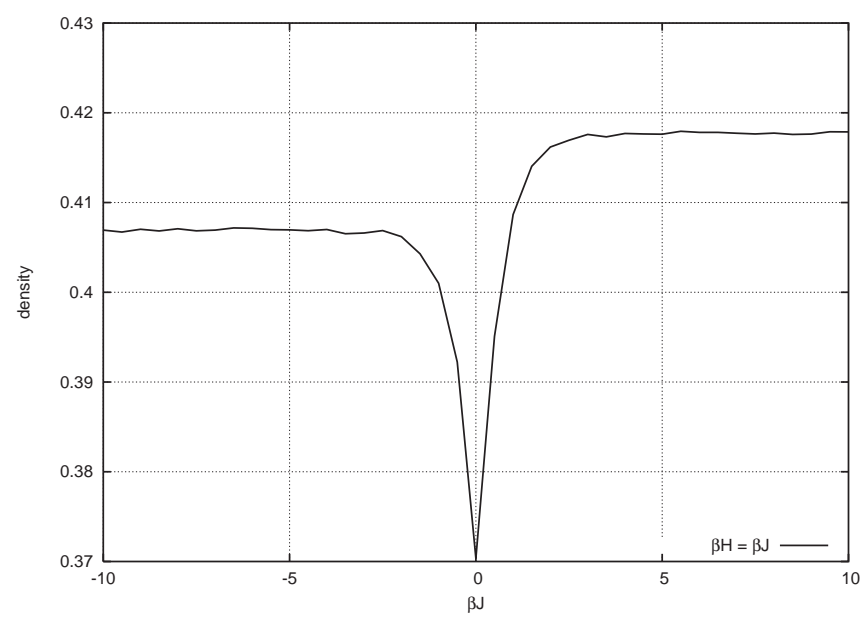

Fig. 9. Dependency of the density on $\beta J$ for $\beta H=\beta J$, i.e., the deepest trench.

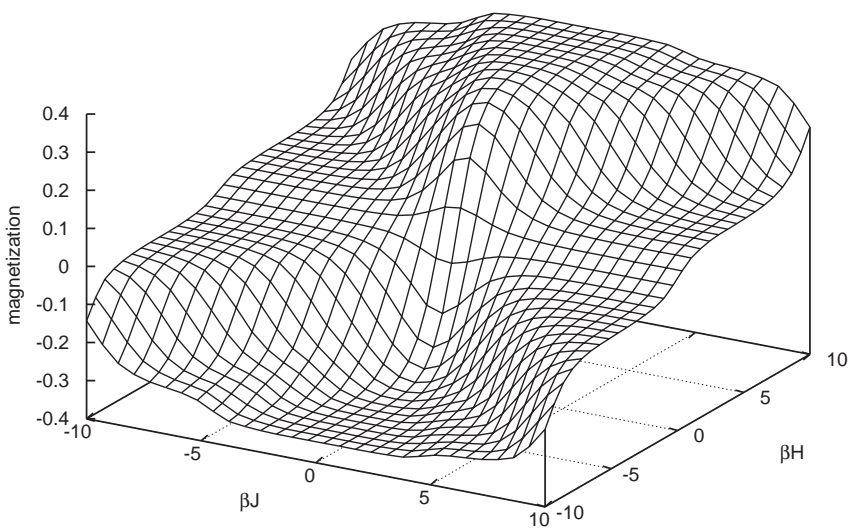

Fig. 10. Dependence of the magnetization on $\beta J$ and $\beta H$.

where $n_{+}$and $n_{-}$are the number of up and down spins, respectively. This quantity can be considered as a measure of the difference in grain orientations in the packing.

As seen in Fig. 10, the magnetization dependence on $\beta H$ and $\beta J$ presents a sort of terraces and is slightly undulating, at the borders of specific regions previously emphasized in the density dependence discussion. Interestingly, all expected dependences are better visible on the magnetization than on the density pictures. In particular, see Fig. 11.

Figs. 12 and 13 present the behavior of the magnetization for different $\beta J$ and $\beta H$ values. The maximum magnetization occurs for $\beta J=0$ and no field. For a finite field the maximum is rather broad. Terrace structures are seen like in Fig. 10 for the density. There are about six terraces with different values of the magnetization. Each terrace 


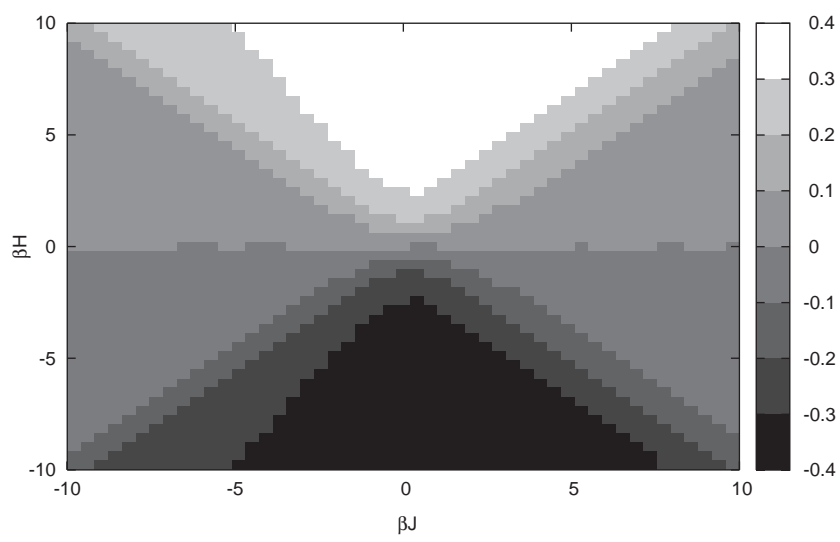

Fig. 11. Dependence of the magnetization on $\beta J$ and $\beta H$, with a gray color scale as indicated.

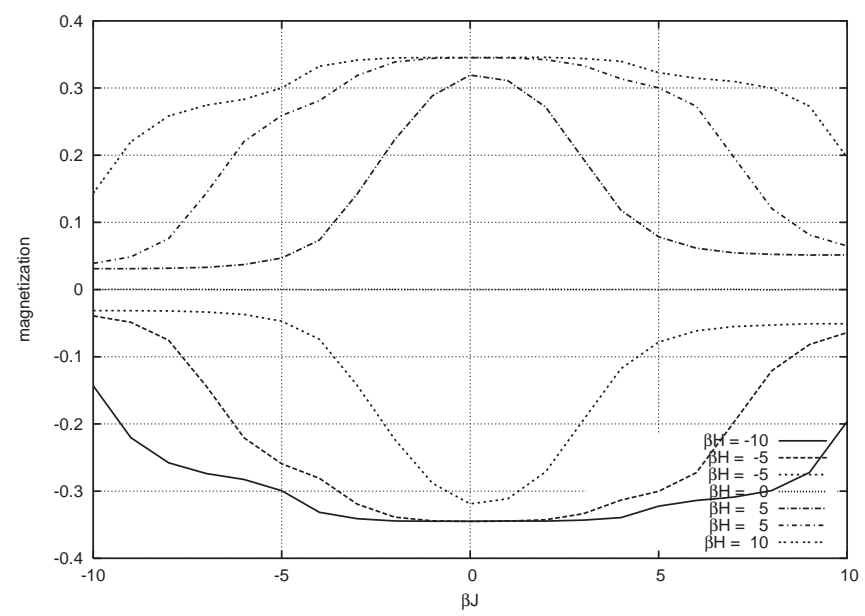

Fig. 12. Dependence of the magnetization on $\beta J$ for different values of $\beta H$.

occurs in ranges like those of the regions previously mentioned. It is emphasized that the magnetization terrace levels differs in the antiferromagnetic and the ferromagnetic coupling regions, i.e., for $\beta J<0$ and $\beta J>0$,- the level height depending on the field sign.

This is well stressed through Fig. 13 which exhibits the dependence of the magnetization on $\beta H$ for different $\beta J$. Notice that for the case without spin-spin interaction, one obtains a kind of saturation at a value ca. \pm 0.36 . Observe the surprising form of the $(M, H)$ curve, as for classical soft magnets. For a finite field, the magnetization does not saturate like in the case of zero field, for the values that we have investigated, but $M$ saturates creating stair-like structures. 


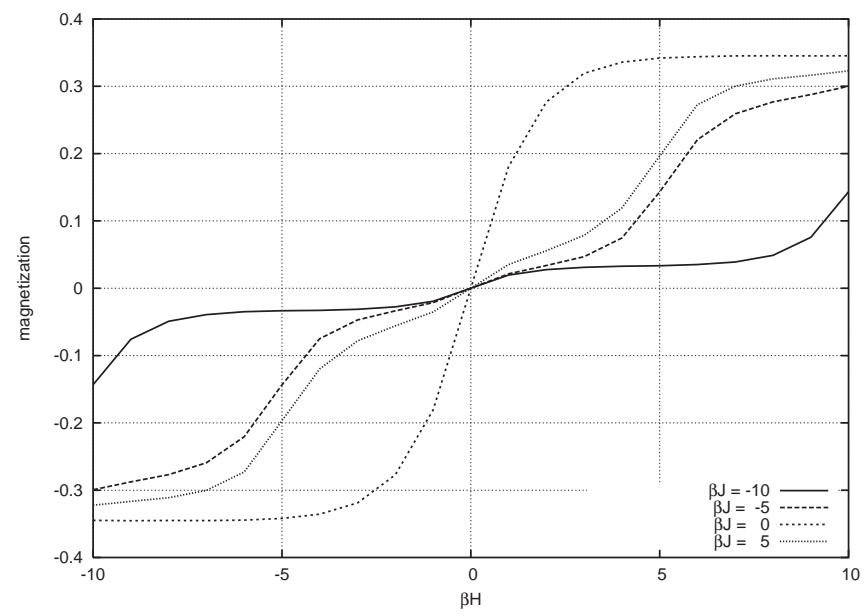

Fig. 13. Dependence of the magnetization on $\beta H$ for different $\beta J$ values.

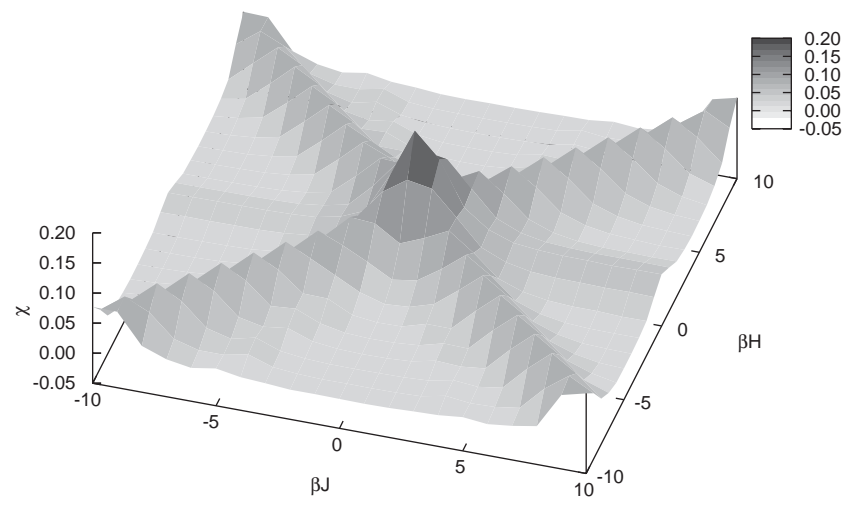

Fig. 14. Dependence of the magnetic susceptibility on $\beta J$ and $\beta H$.

\subsection{Magnetic susceptibility}

The magnetic susceptibility of the clusters can be obtained by numerically differentiating the magnetization $M$ over the field $H$, at fixed $J$ or $H$, i.e.

$$
\chi_{J}=\left.\frac{\mathrm{d} M}{\mathrm{~d} H}\right|_{J}, \quad \chi_{H}=\left.\frac{\mathrm{d} M}{\mathrm{~d} J}\right|_{H} .
$$

The dependence is presented in Fig. 14. The highest susceptibility occurs for regions where $\beta H=\beta J=0$ and the main trenches. Other regions have a rather relatively small susceptibility. The results can be understood through the role of the interaction between spins which as usual induces a drop in the magnetic susceptibility of the materials. On the other hand, the variation of the orientation difference $(M)$ of the grains in a 


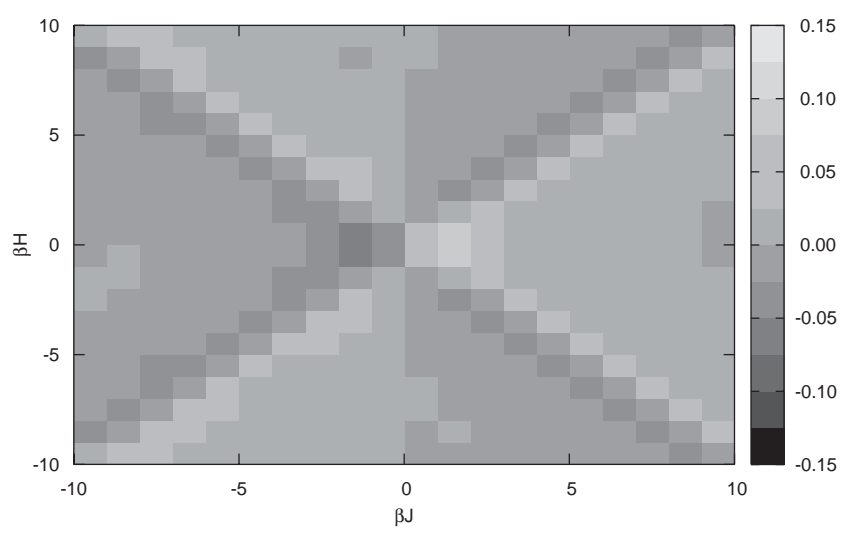

Fig. 15. Dependence of the compressibility on $\beta J$ and $\beta H$.

packing with respect to an external (wind or) field should be an interesting experimental test.

\subsection{Compressibility}

Similarly, the compressibility (Fig. 15) at fixed $H$ or $J$ is

$$
\kappa_{H}=-\left.\frac{1}{G} \frac{\mathrm{d} G}{\mathrm{~d} J}\right|_{H}, \quad \kappa_{J}=-\left.\frac{1}{G} \frac{\mathrm{d} G}{\mathrm{~d} H}\right|_{J},
$$

where $G$ is the density. The displayed data is rather blurred because of the limited amount of data for numerical differentiation near the trenches and in the region of the standard BD, in particular. Nevertheless, this figure indicates some mild variation due to internal competition and external conditions. Let us also recall that while the susceptibility is singular in spin glass models of compaction [32] at the critical percolation value, the compressibility seems to remain finite. [32,33] Our model indicates the same, as experimentally or numerically observed. Further experimental and numerical considerations should be given to this point.

\section{Pile structures}

In this section we present some examples of typical clusters created by the MBD in specific regions, as observed and discussed here above. The size of the lacunes in each cluster allows some emphasis and contrasting.

\subsection{Typical clusters}

In Fig. 16, nine clusters from nine different growth regions are shown. The central cluster has the smallest density: it corresponds to the case when there is no interaction between spins and no field, i.e. it is the standard BD model [26]. 


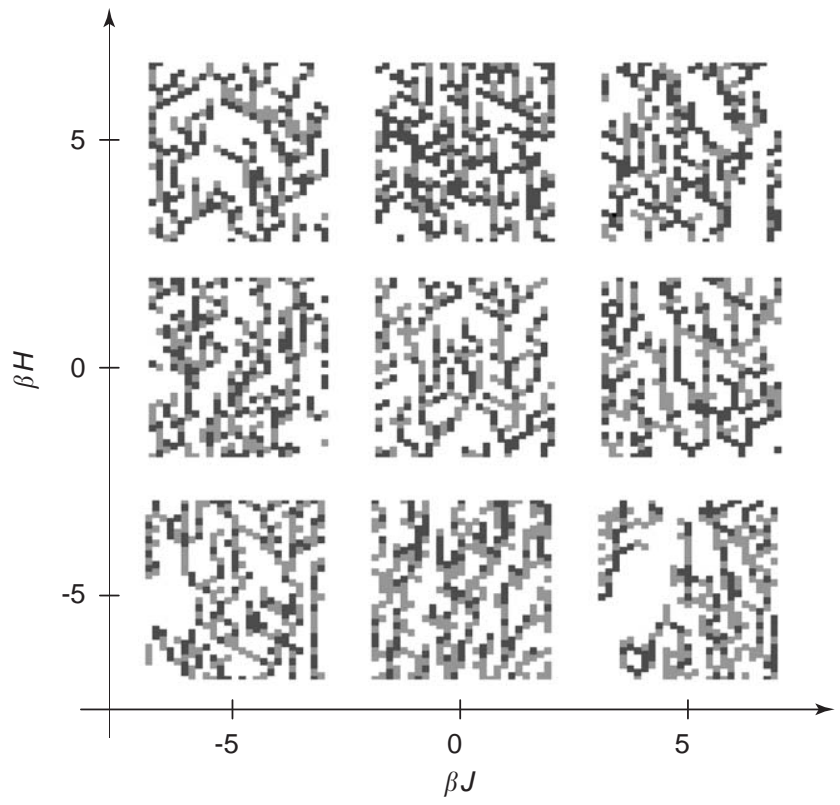

Fig. 16. Typical clusters. The "colors" correspond to the spin's sign.

On the other hand the clusters for $\beta J=0$ and $\beta H= \pm 5$ have the highest density; they illustrate regions where the density is saturating (above 0.44 , see Fig. 3 ). It should be mentioned that the results are symmetrical with respect to the field sign.

For a finite $J$ interaction between spins, a quite different behavior of the clusters is observed. The clusters corresponding to the trenches $(\beta J= \pm 5$ and $\beta H= \pm 5)$ have a smaller density (ca. 0.41) than those outside the trenches (see for example clusters with $\beta J=0$ and $\beta H= \pm 5$ ). Also observe, in Fig. 16, field-free grown clusters and differences between $\beta J<0$ and $\beta J>0$ clusters. When $\beta J>0$, the spins show a tendency toward similar sign spin "domains". In the antiferromagnetic-like region, i.e. where $\beta J<0$, adjacent spins have more often opposite directions - a cluster with $\beta J<0$ and $\beta H=0$ is a typical example. This allows us to emphasize that the internal competition leads to different cooperative phenomena in cluster packing [3].

\subsection{Fractal dimension}

For further relating the model and our investigations to granular piles, it is of interest to check the fractal dimension $[34,35]$ of the piles in the different parameter regions. The (box counting) technique [34,35] consists in covering, without overlapping, the whole cluster by squares of the same size, and computing the number of squares which have at least one spin up (or down), for different square sizes. We have distinguished between the fractal dimension of the cluster of up and down spins. When computing the fractal dimension of the whole cluster it was simply checked whether there was at 


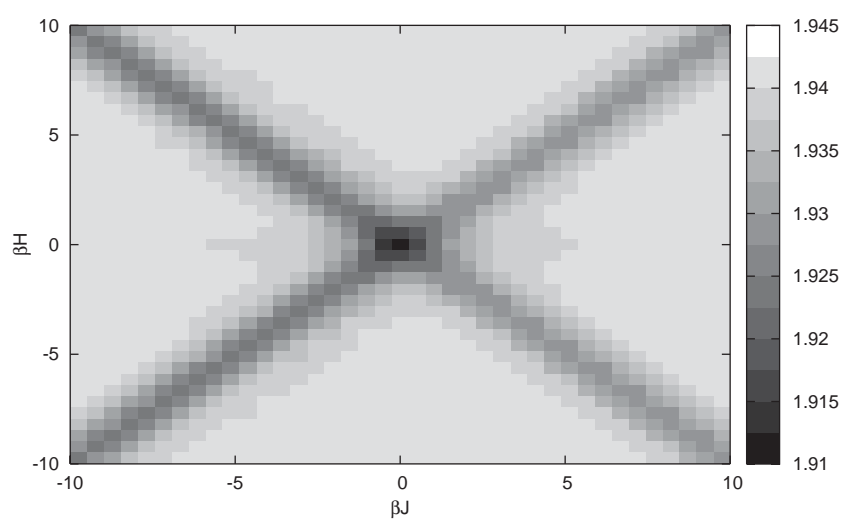

Fig. 17. The fractal dimension for the whole cluster, i.e. not recognizing the spin signs.

least one spin and its sign in the relevant square. From the best linear fit to the data, i.e., $-\log ($ square size $)$ vs. $\log$ (number of squares with a spin), the fractal dimension is obtained through the slope. The results are reported in Figs. 17 and 18.

Let us discuss the results corresponding to different regions, either not distinguishing over the spin sign (Fig. 17), or on the contrary considering down or up cases (Fig. 18a or b, respectively). The fractal dimension in every case ranges from 1.91 to 1.95 , therefore is about equal to 2 , taking into consideration the error bars. This value is similar to what is found in classical BDM [4,26] and in the rain model [25]. Surprisingly, the lowest fractal dimensions are found in the above for the trenches and in particular for the classical BDM.

\section{Conclusion}

We have presented a nonequilibrium ballistic deposition model with one degree of freedom per entity, degree which can be coupled to an external field. We have examined the cluster properties emphasizing the existence of two-order parameters, since two characteristic field $(J$ and $H$ ) are intrinsic to the model. This model can serve to describe in a first approximation the deposition of a distribution of grains, distribution characterized by one intrinsic parameter which can be coupled to an external field. The degree of freedom can be either the anisotropy factor or the surface roughness or an electrostatic imbalance of a grain - the corresponding field being immediately thought of.

For the sake of such an extension of usual deposition models, the degree of freedom has been called "spin". We have simulated the nonequilibrium deposition in a finite size $2 \mathrm{D}$ vertical box, admitting that grains flow down along linear trajectories on a triangular lattice. The "quenching" of the degree of freedom on the cluster leads to branching or compactness and moreover to combined geometric and physical regions at specific "field" and "spin-spin interaction" values. This was seen through the calculation of the 

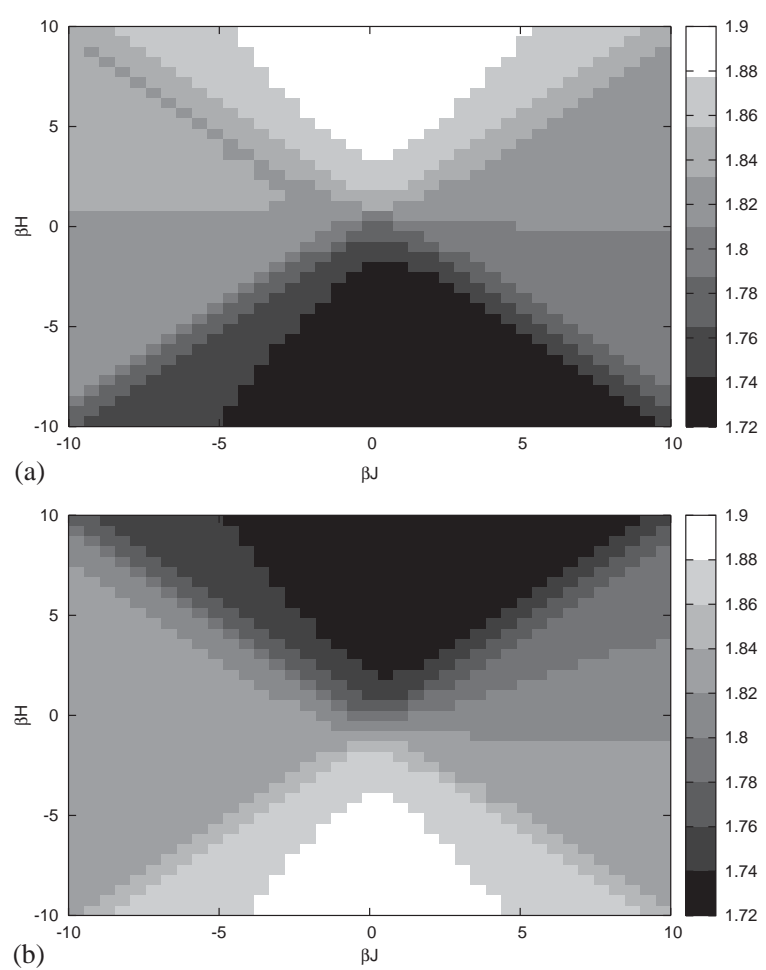

Fig. 18. The fractal dimension for the granular cluster when we recognize the spin signs.

"density" and the so called "magnetization". Different cluster regimes were expected according to the (spin sticking) packing rule.

We have investigated a box geometry and a triangular underlying lattice. The ranges in density and magnetization are limited, but features exist resulting from competitive nonequilibrium growth/deposition processes. Minima in density occur along specific sticking probability lines. Slight differences exist whether the "spin-spin interaction energy" is positive or negative. The fractal dimension of clusters whatever the type of grains and the parameter sign or values is however rather trivial and equal to 2 . This differs markedly from what was found in the MDLA model starting from a central seed in which both in the ferromagnetic interaction regions, and the AF regions, the cluster morphology was dendritic with an important thickening of the branches and the fractal dimension ranging from 1.68 to 1.99 . Instead of a critical a value $\beta J_{c}$, a set of values depending on the external field divide the parameter plane in regions, with trenches and plateaus. Thus, a spreading phenomenon is avoided around such $\beta J_{c}(H)$. It is of interest to further examine whether this has interesting consequences in granular deposition situations [36].

The connection between this model and granular matter systems suggests some experimental work. In the MBD the spin can be interpreted as a rotation or defining a 
direction process. The coupling constant can be mapped to a mechanical friction energy, the magnetic field to gravity or wind pressure. It is known that packing is impaired by static electrical charges. Such effects may be considered in the above framework.

\section{Acknowledgements}

KT is supported through an Action de Recherche Concertée Program of the University of Liège (ARC 02/07-293). Comments by R. Cloots, L. Delattre, A. Pȩkalski, $\mathrm{N}$. Vandewalle and other coworkers of the ARC program on granular materials at ULg are greatly appreciated.

\section{References}

[1] H. Herrmann, Grains of understanding, Phys. World 10 (1997) 31;

H. Herrmann, S. Luding, Modeling granular media on the computer, Continuum Mech. Thermodyn. 10 (1998) 189;

H.J. Herrmann, J.-P. Hovi, S. Luding, Physics of Granular Media, Kluwer, Dordrecht, 1998.

[2] R.B. Pandey, D. Stauffer, R. Seyfarth, L.A. Cueva, J.F. Gettrust, W. Wood, Density profile and flow of miscible fluid with dissimilar constituent masses, Physica A 310 (2002) 325.

[3] J. Faraudo, New cooperative effects in ballistic deposition of hard disks, Phys. Rev. Lett. 89 (2002) 276104.

[4] H.J. Herrmann, Geometrical cluster growth models and kinetic gelation, Phys. Rep. 136 (1986) 153.

[5] R. Jullien, R. Botet, Scaling properties of the surface of the Eden model in $D=2,3$, and 4, J. Phys. A 18 (1985) 2279.

[6] T.A. Witten, M.L. Sander, Diffusion-limited aggregation, a kinetic critical phenomenon, Phys. Rev. Lett. 47 (1981) 1400.

[7] Z. Zhang, X. Chen, M.G. Lagally, Bonding-geometry dependence of fractal growth on metal surfaces, Phys. Rev. Lett. 73 (1994) 1829.

[8] N. Vandewalle, R. Cloots, M. Ausloos, Simulated growth and microstructure of $\mathrm{DyBa}_{2} \mathrm{Cu}_{3} \mathrm{O}_{7-x}$ with and without $\mathrm{Dy}_{2} \mathrm{BaCuO}_{5}$ addition, J. Mater. Res. 10 (1995) 268;

N. Vandewalle, M. Ausloos, R. Cloots, The kinetic growth anisotropy of the 123-(RE)BaCuO compounds, Philos. Mag. A 72 (1995) 727.

[9] H. Abbey, An examination of the Reed Frost theory of epidemics, Hum. Biol. 24 (1952) 201.

[10] H.J. Herrmann, S. Roux, Statistical Models for the Fracture of Disordered Media, North-Holland, Amsterdam, 1990;

M. Ausloos, J.M. Kowalski, Stochastic models of two-dimensional fracture, Phys. Rev. B 45 (12) (1992) 830 .

[11] J. Alonso, J.-P. Hovi, H. Herrmann, A model for the calculation of the angle of repose from microscopic grain properties, Phys. Rev. E 57 (1998) 672.

[12] K. To, P.-Y. Lai, H.K. Pak, Jamming of granular flow in a two-dimensional hopper, Phys. Rev. Lett. 86 (2001) 71;

N. Pieret, R. Mahnke, M. Ausloos, N. Vandewalle, Stochastic master equation approach to aggregation in freeway traffic, in: M. Schreckenberg, D.E. Wolf (Eds.), Traffic and Granular Flow 97, Springer, Singapore, 1998, pp. 447-452.

[13] J. Duran, E. Kolb, L. Vanel, Static friction and arch formation in granular materials, Phys. Rev. E 58 (1998) 805 .

[14] R. Potts, Some generalized order-disorder transformations, Proc. Cambridge Philos. Soc. 48 (1952) 106

[15] S.N. Majumdar, D.S. Dean, Slow relaxation in a constrained Ising spin chain: a toy model for granular compaction, Phys. Rev. E 66 (2002) 056114. 
[16] M. Ausloos, N. Vandewalle, R. Cloots, Magnetic kinetic growth models, Europhys. Lett. 24 (1993) 629

M. Ausloos, N. Vandewalle, R. Cloots, Magnetic kinetic growth models, J. Magn. Magn. Mater. 140-144 (1995) 2185.

[17] N. Vandewalle, M. Ausloos, Magnetic diffusion-limited aggregation, Phys. Rev. E 51 (1995) 597;

N. Vandewalle, M. Ausloos, Growth models with internal competition, Acta Phys. Pol. B 27 (1996) 737.

[18] R. Pastor-Satorras, J.M. Rubi, Particle-cluster aggregation with dipolar interactions, Phys. Rev. E 51 (1995) 5994;

R. Pastor-Satorras, J.M. Rubi, Ballistic adsorption of colloidal magnetic particles, Comput. Phys. Comm. $121-122$ (1999) 265.

[19] F. de los Santos, M. Tasinkevych, J.M. Tavares, P.I.C. Teixeira, Deposition of magnetic particles: a computer simulation study, J. Phys. C: Cond. Matter 15 (2003) 1291.

[20] J. Candia, E.V. Albano, Comparative study of an Eden model for the irreversible growth of spins and the equilibrium Ising model, Phys. Rev. E 63 (2001) 066127.

[21] Cl. M. Horowitz, R.A. Monetti, E.V. Albano, Competitive growth model involving random deposition and random deposition with surface relaxation, Phys. Rev. E 63 (2001) 066132.

[22] J. Candia, E.V. Albano, Irreversible growth of a binary mixture confined in a thin film geometry with competing walls, Phys. Rev. Lett. 88 (4) (2002) 016103.

[23] M. Mueller, K. Binder, E.V. Albano, Finite size effects on the phase diagram of a binary mixture confined between competing walls, Physica A 274 (2000) 188.

[24] J. Candia, E.V. Albano, Non-equilibrium wetting transition in a magnetic Eden model, Eur. Phys. J. B 16 (2000) 531.

[25] W. Wang, H.A. Cerdeira, Kinetic growth of randomlike and ballisticlike deposition models, Phys. Rev. E 47 (1993) 3357;

H.F. El-Nashar, H.A. Cerdeira, Dynamic scaling in a ballistic deposition model for a binary system, Phys. Rev. E 61 (2000) 6149;

H.F. El-Nashar, W. Wang, H.A. Cerdeira, Growth morphology for a ballistic deposition model for multiple species, Phys. Rev. E 58 (1998) 4461.

[26] P. Meakin, R. Jullien, Simple ballistic deposition models for the formation on thin films, SPIE 48 (1987) 1651;

F. Family, T. Vicsek, Scaling of the active zone in the Eden process on precolation networks and the ballistic depositon model, J. Phys. A 18 (1985) L75;

R. Baiod, D. Kessler, P. Ramanlal, L. Sander, R. Savit, Dynamical scaling of the surface of finite-density ballistic aggregation, Phys. Rev. A 38 (1988) 3672.

[27] P. Meakin, P. Ramanlal, L.M. Sander, R.C. Ball, Ballistic deposition on surfaces, Phys. Rev. A 34 (1986) 5091.

[28] S.S. Manna, Two-state model of self-organized criticality, J. Phys. A. Math. Gen. 24 (1991) L363.

[29] R. Dickman, T. Tom, M.J. de Oliveira, Sandpiles with height restrictions, Phys. Rev. E 66 (2002) 016111.

[30] M. Blume, V.J. Emery, R.B. Griffiths, Ising model for the Lambda transition and phase separation in $\mathrm{He}_{3}-\mathrm{He}_{4}$ mixtures, Phys. Rev. A 4 (1971) 1071.

[31] M. Ausloos, P. Clippe, J.M. Kowalski, A. Pekalski, Magnetic lattice gas, Phys. Rev. A 22 (1980) 2218.

[32] M. Nicodemi, A. Coniglio, H.J. Herrmann, The compaction in granular media and frustrated Ising models, J. Phys. A. Math. Gen. 30 (1997) L379.

[33] J.J. Arenzon, M. Nicodemi, M. Sellitto, Equilibrium properties of the Ising frustrated lattice gas, J. Phys. I (France) 6 (1996) 1143.

[34] M. Schroeder, Fractals, Chaos and Power Laws, W.H. Freeman and Co., New York, 1991.

[35] B.J. West, B. Deering, The Lure of Modern Science, World Scientific, Singapore, 1995.

[36] Z.T. Chowhan, Segregation of Particulate Solids, Pharm. Technol. 19 (1995) 56. 